\title{
Towards a Framework Empowering Software Testing Process for Better Quality
}

\author{
Osama M. Abu EInasr \\ College of Computers and \\ Information Sciences, \\ Egypt.
}

\author{
Magdy Z. Rashad \\ College of Computers and \\ Information Sciences, Egypt.
}

\author{
Mohammed A. Abo- \\ Elsoud \\ College of Computers and \\ Information Sciences, \\ Egypt.
}

\author{
Gamal M. Aly \\ College of Engineering, \\ Egypt.
}

\begin{abstract}
Software development frameworks are considered the engine that leads the development activities within software development organizations, so the main interest in this research is establishing a framework to effectively lead the development activities to enhance the software quality. The proposed framework relates each development phase with its available artifacts, software metrics can be extracted from its artifacts and relates the test practices and QA best practices during the SDLC. It provides an organized approach to handle miscommunication and poor team management that usually lead to team confusion. It also provides a way to disengage the ambiguity between the software fault and failure through establishing a clear relationship between the both concepts and developing automated models to predict the software correctness and measure its reliability.
\end{abstract}

Keywords

SDLC, QA, Static Analysis, Testing, Fault, Failure, Correctness, Reliability.

\section{INTRODUCTION}

Software development organizations seek to improve the quality of their software by continuous working to adapt their development models. Some still depend entirely on the traditional model and others moving strongly towards the agile methodologies with closed eyes about the advantages and disadvantages of the both models, despite their influence on the pillars of the software development process. There are great attempts in the research environment to bring the views and to create models combine the advantages of each model [1-5], other attempts seek to automate the verification activities in order to get a complete control of the behavior of the software and its related quality attributes [6-10].

Software measurement analysis, fault prediction and failure estimation techniques become rich area of research related to verification activities. By evaluating the attributes of the software, we can know its status, characteristics and behavior, [11,12]. The fault proneness [13-16], defect density [16-19], and failure radiation information [20] provide important guidelines to testing practitioners to prioritize their testing effort and assign verification and validation activities.

Most studies related to the research area are considered individual contributions that seek to automates some of the software development activities without concern for creating a framework that includes those contributions to building an integrated framework can be relied upon to manage the three elements of the development system; people, process and product [21-25]. The paper extends the work done by $\mathrm{Yu}$ BengLeau [1] and Milad Hanna [10]. Yu BengLeau [1] has been introduced a review paper that explains the advantages and disadvantages of both traditional and agile methodologies and suggests improvements for current agile development for enhancing organizational project management. Milad Hanna [10] has been introduced a new scripting technique that facilitates the process of automating the execution phase through software testing in an industrial context.

The paper seeks to provide a framework that manages the stakeholders and draws the relationship between each process within the various phases of the system and the deliverables of them with clarifying entity responsible for the production and the beneficiary of its existence in order to structure the inner workings of the system. It sheds light on the pivotal role played by the applications of artificial intelligence to automate the software fault prediction process in a manner of errors spread, their location, and the extent of their impact on the development process and their related quality attributes. It also gives a broad interest to study the behavior of the software product through analyzing and reviewing the deliverables of each phase using what are known by the term software metrics which are used as a basis to build a system capable of monitoring the correctness of software product and its reliability.

The remainder of this paper is organized as follows; Section 2 highlights the problems associated with current area of research. Section 3 provides the full description of the proposed framework. Section 4 introduces the conclusion and the future work.

\section{PROBLEM DESCRIPTION}

In spite of the successive efforts to develop models of development, but it still suffers from foggy specification of the basic development components of the system ; people, process and product. There are still issues concerning the management of stakeholders, not to mention chaotic the overlapping among the development processes and their integration with each other to produce cost effective qualified software product, and other drawbacks such as:

- Lack of a conceptual framework that provide a clear distinction between software fault and failure, their causes, effects and their prediction and estimation models.

- Lack of an organized approach to handle miscommunication and poor team management that usually lead to team confusion.

- Lack of the distinction between static analysis activities and dynamic testing activities and their scope in handling software quality. 
- Lack of a conceptual specification of the tasks of each development process, its interest, and its deliverables.

- Fogginess to obtain information about the progress of the development process until the late stages of the age of the system.

It becomes very important to develop a conceptual framework that brings the views between traditional development activities, agile practices and their related quality activities to get rid of the above mentioned challenges.

\section{PROPOSED FRAMEWORK}

The proposed framework relates each development phase with its available artifacts, software metrics can be extracted from its artifacts and relates the test practices and QA best practices during the SDLC.

Section 3.1 introduces the framework architecture and its stages. Section 3.2 introduces the four classification dimensions of the framework activities. Section 3.3 introduces the project organizational structure and the team formation and their roles. Finally, Section 3.4 introduces the full description of the framework's phases their activities and their classification.

\subsection{Framework Architecture}

The framework architecture comprised into three distinct interleaved stages; the Pre-Development, Development and Post-Development stage. These three stages have a number of internal phases. Fig. 1 shows the framework components.

3.1.1 Software Pre-Development stage assure that the project commitments have been clearly defined considering a set of activities related to the project vision, resources required, the schedule and budget, project risk handling, business requirement and various project management plans through initiation and the planning phases.

3.1.2 Software development stage decomposed into six distinct, often overlapped / interleaved phases; requirement, design (High Level Design and Low Level Design), code and unit test, Pre-Testing, Test Execution (Higher Order Test) and Post-testing phases. For each phase in the development stage the possible created artifacts have been introduced to be able to monitor, review, predict and measure the progress of work done through these phases and assuring the quality of the produced release.

3.1.3 Software Post-Development stage concerned with managing the implementation of the produced release in the operational environment through deployment and operation phase.

\subsection{Activities Classification's Dimensions}

Software activities can be classified along the proposed framework into four dimensions; Management, Development, Review and Prediction and / or Estimation sector.

3.2.1 Management sector involves the development of project management plans (PMP) that provide a great control of the development activities and the introduction of managerial support actions that mainly prevent or minimize schedule and budget failures, continually changes in user requirements and identify and assess the risks resulting from the change one of the elements responsible for the software production through the various stages of the system.

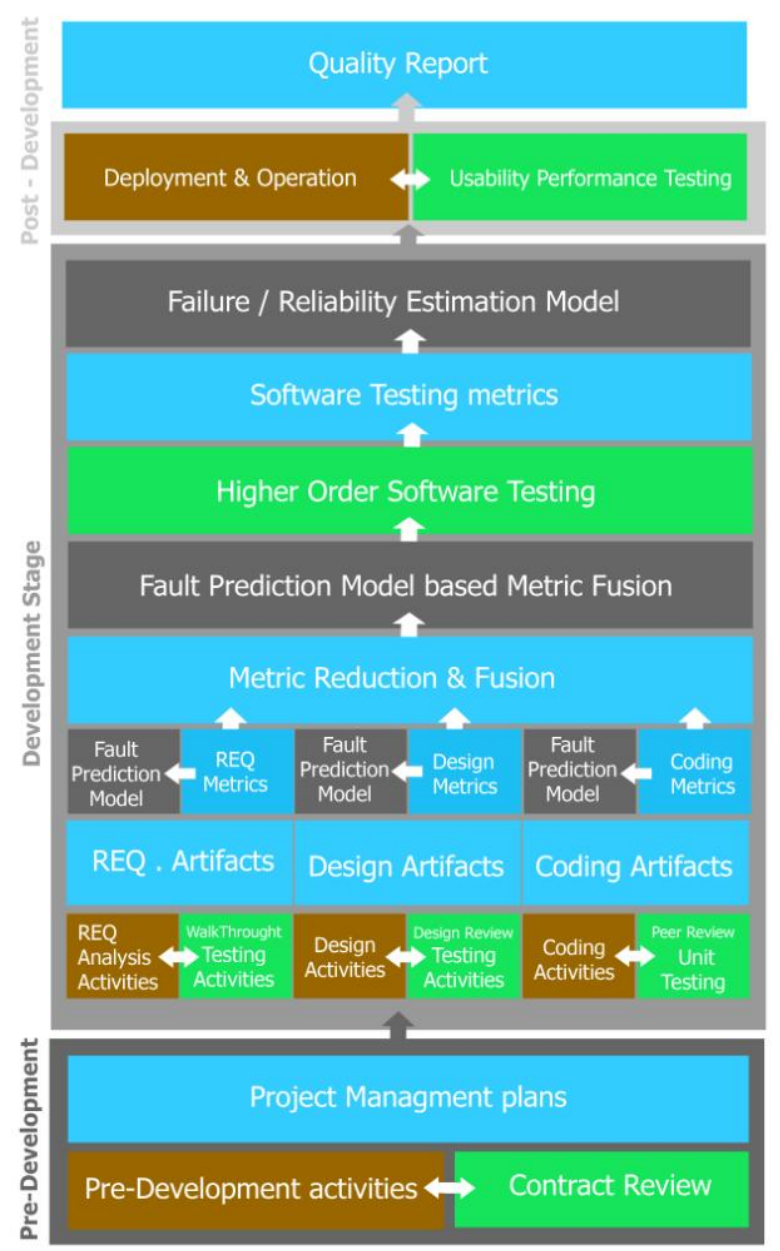

Fig 1: Framework Architecture.

3.2.2 Development sector includes a set of processes that concerned with developing the various deliverables of the release / product and managing their delivery to review sector for auditing and reviewing its quality and be ready for redeveloping or modifying its construction until it reaches the desired level of quality.

3.2.3 Review / Measurement Analysis sector includes a range of verification activities such as walkthrough, design review, peer review and inspection. It also locates the associated metrics for each software artifact that reflect the behavior of the software created through the development stage.

3.2.4 Prediction/ Estimation sector includes the construction of a range of models designed to empower the software testing process for improving the quality of the product and so by focusing on reducing the effort required to determine the presence or eliminate errors in product or assess the readiness of the product for use.

During the prediction part, fault prediction models that predict either fault-prone modules or fault content of the software modules based on collected metrics has been established. The failure estimation part of this sector attempts to estimate the current level of reliability of the software while in execution and determines the readiness of the system to release. Thus, the number of failures becomes the goal of estimation instead of faults. Fig.2 shows the classification of the fault prediction and estimation models and their implementation techniques. 


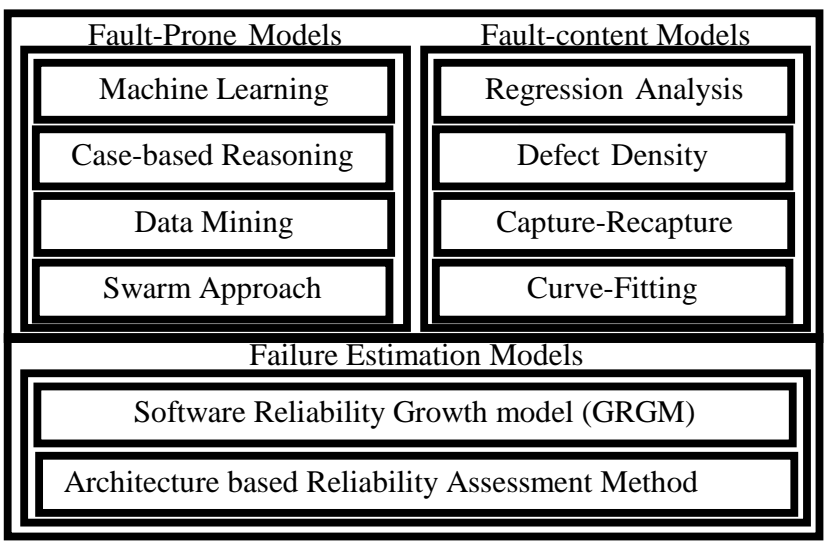

Fig 2: Fault prediction and failure estimation models and their implementation techniques.

\subsection{Stakeholders Involvement}

During each stage within the proposed framework, several stakeholders may be participated in the software project. A stakeholder is someone who has interest in the success of the project. Depending on their roles, different stakeholders may have different interests in the software project and involved in different software teams, such as; Planning, Dev, Testing, and System team. Table 1 illustrates the most typical project organizational structure and teams' formation.

Table 1. Project organizational structure and Teams' Formation

\begin{tabular}{|c|c|c|c|c|c|c|c|}
\hline \multirow[b]{2}{*}{ Stakeholder } & \multicolumn{4}{|c|}{ Team Involvement } & \multicolumn{3}{|c|}{ Interest } \\
\hline & 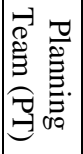 & 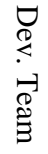 & 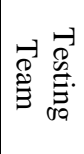 & 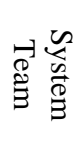 & $\begin{array}{l}\text { r. } \\
0 \\
0 \\
0 \\
0 \\
0\end{array}$ & 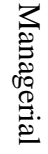 & 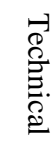 \\
\hline Customer & $\checkmark$ & & & & - & & \\
\hline User & $\checkmark$ & $\checkmark$ & $\checkmark$ & $\checkmark$ & - & & \\
\hline PM & $\checkmark$ & $\checkmark$ & $\checkmark$ & $\checkmark$ & - & $\cdot$ & \\
\hline HRM & $\checkmark$ & & & & & - & \\
\hline $\begin{array}{l}\text { Master } \\
\text { Developer }\end{array}$ & $\checkmark$ & $\checkmark$ & $\checkmark$ & & & - & - \\
\hline $\begin{array}{l}\text { S.W } \\
\text { Developer }\end{array}$ & & $\checkmark$ & & & & & - \\
\hline Master Tester & $\checkmark$ & $\checkmark$ & $\checkmark$ & $\checkmark$ & & . & . \\
\hline Tester & & & $\checkmark$ & & & & - \\
\hline QA Lead & $\checkmark$ & & $\checkmark$ & $\checkmark$ & - & - & \\
\hline $\begin{array}{l}\text { System } \\
\text { Engineer }\end{array}$ & $\checkmark$ & & $\checkmark$ & $\checkmark$ & & - & - \\
\hline $\begin{array}{l}\text { Technical } \\
\text { Support }\end{array}$ & & & & $\checkmark$ & & & - \\
\hline
\end{tabular}

\subsection{Full description of the framework's \\ phases}

The following section explains in details the full description of the framework's activities. For each phase of the framework, the phase purpose followed by phase processes, their classification, and their associated artifacts have been established.

\subsubsection{Initiation Phase}

It concerned with the project vision, resource, and scope. Its main purpose is studying the feasibility of the project and initiating the project request. Table 2 illustrates the initiation phase's processes, their classification, and their associated artifacts.

\subsubsection{Planning Phase}

It concerned with the development of Project Management Plans (PMP) and establishing various planning activities.

Table 3 illustrates the planning phase's processes, their classification, and their associated artifacts.

\subsubsection{REQ. Analysis Phase}

It concerned with producing the requirement documents both user and system requirements and its related testing artifacts. Once these artifacts have been documented, the review process done using walkthrough approach to produce the requirement metrics that used as basis for feeding the fault prediction model. Table 4 illustrates the REQ. analysis phase's processes, their classification, and their associated artifacts.

\subsubsection{Design Phase}

It concerned with the development of System Design Document (SDD) and its related testing artifacts such as unit and integration testing templates. Once the prototype has been created, the review process done using design review that updates static analysis report and produce design metrics used as basis for feeding the fault prediction model. Table 5 illustrates the design phase's processes, their classification, and their associated artifacts.

\subsubsection{Coding Phase}

During the coding phase the creation of the software product is underway and parts of the software product, such as classes, modules and subsystems are created. Unit testing represent a dynamic testing approach that test individual components of the system independently. Once peer review activities have been conducted, code metrics used as basis for feeding the fault prediction model are generated. Table 6 illustrates the coding phase's processes, their classification, and their associated artifacts.

\subsubsection{Pre-Testing Phase}

During the Pre-Test phase, Test case reduction has been conducted to select the most obvious test cases, and construct the software metrics repository that feed the fault prediction model that used mainly for managing the testing efforts. Table 7 illustrates the pre-testing phase's processes, their classification, and their associated artifacts.

\subsubsection{Testing Execution Phase}

After testing the individual components, a higher order test focus on the system as a whole and conducted as a series of testing issues that test the system from several perspectives including; Integration Test, Release Test, and Security Test. Table 8 illustrates the testing phase's processes, their classification, and their associated artifacts.

\subsubsection{Post-Testing Phase}

It concerned with assessing the readiness of the product to be released. Table 9 illustrates the post-testing phase's processes, their classification, and their associated artifacts.

\subsubsection{Deployment and Operation Phase}

It concerned with installing release into production environment, supporting its operation, reviewing its security and monitoring its performance. Table 10 illustrates the deployment and operation phase's processes, their classification, and their associated artifacts. 
Table 2. Initiation Phase's processes, their classification, and associated artifacts

\begin{tabular}{|c|c|c|c|c|c|c|c|}
\hline \multirow[t]{2}{*}{ Initiation Processes } & \multicolumn{4}{|c|}{ Classification } & \multirow[t]{2}{*}{ Artifact } & \multicolumn{2}{|c|}{ Roles } \\
\hline & 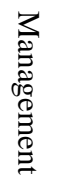 & 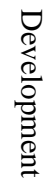 & 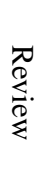 & 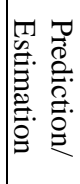 & & 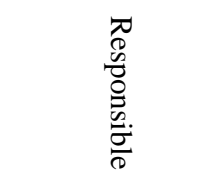 & 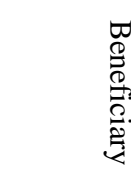 \\
\hline Initiate Business Case \& Scope & - & & & & PSD & PM, Customer & PT \\
\hline Establish Team Structure & - & & & & organizational chart & PM, HRM & $\mathrm{PT}$ \\
\hline Identify Preliminary Risks & - & & & & Risk Assessment & PM & PT \\
\hline Establish project Proposal & - & & & & Project Proposal & PM, Customer & PT \\
\hline Establish Project Charter & - & & & & Project Charter & PT & PT \\
\hline Contract Review & & & - & & Feasibility Report & PM, Customer & PT \\
\hline Phase Status Review & & & - & & Status Report & PM & PT \\
\hline
\end{tabular}

Table 3. Planning Phase's processes, their classification, and its artifacts

\begin{tabular}{|c|c|c|c|c|c|c|c|}
\hline \multirow[t]{2}{*}{ Planning Processes } & \multicolumn{4}{|c|}{ Classification } & \multirow[t]{2}{*}{ Artifacts } & \multicolumn{2}{|c|}{ Roles } \\
\hline & 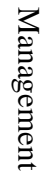 & 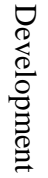 & 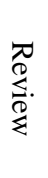 & 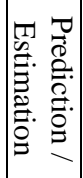 & & $\begin{array}{l}\overrightarrow{0} \\
0 \\
\dot{0} \\
0 \\
0 \\
\frac{0}{0} \\
\frac{\sigma}{0}\end{array}$ & 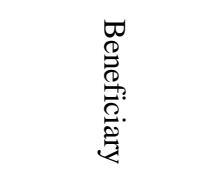 \\
\hline Establish WBS & - & & & & WBS & PM & PT \\
\hline Define Detailed Roles & - & & & & RAM & PM, HRM & PT \\
\hline Estimate Efforts & - & & & & $\begin{array}{l}\text { Staff Plans } \\
\text { Resource Plans }\end{array}$ & PM, HRM & PT \\
\hline Scheduling & - & & & & Schedule Plans & PM & PT \\
\hline Develop PMP & - & & & & PMP & PT & All Stakeholders \\
\hline Phase Status Review & & & - & & Status Report & $\mathrm{PM}$ & PT \\
\hline
\end{tabular}

Table 4. REQ. analysis Phase's processes, their classification, and associated artifacts

\begin{tabular}{|c|c|c|c|c|c|c|c|}
\hline \multirow[t]{2}{*}{ REQ. Processes } & \multicolumn{4}{|c|}{ Classification } & \multirow[t]{2}{*}{ Artifact } & \multicolumn{2}{|l|}{ Roles } \\
\hline & 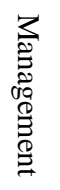 & 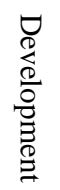 & $\begin{array}{l}\underset{0}{0} \\
\stackrel{0}{<} \\
\stackrel{0}{8} \\
\dot{8}\end{array}$ & 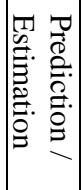 & & 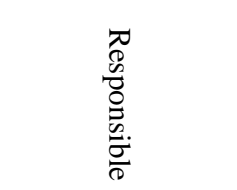 & 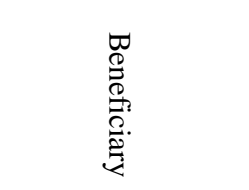 \\
\hline Establish Risk Management & - & & & & Risk Assessment & \multirow[t]{2}{*}{ PM } & \multirow[b]{2}{*}{ PT } \\
\hline Establish Contingency Plans & - & & & & Update PMP & & \\
\hline $\begin{array}{l}\text { Develop Concept Of } \\
\text { Operations }\end{array}$ & & - & & & URD, AUT & Dev Team & All Stakeholders \\
\hline REQ Analysis, Prioritization & & - & & & SRD, RTM & Dev Team & Dev Team \\
\hline MTP formulation & & - & & & MTP & Master Tester & Testing Team \\
\hline REQ Review & & & - & & REQ Metrics, SAR & $\begin{array}{l}\text { QA Lead, Master } \\
\text { Developer }\end{array}$ & Dev, Testing Team \\
\hline Phase Status Review & & & - & & Status Report & PM & PT, Dev Team \\
\hline Prediction based REQ. Metrics & & & & $\cdot$ & TAR & $\begin{array}{l}\text { QA Lead, Master } \\
\text { Tester }\end{array}$ & Testing Team \\
\hline
\end{tabular}


Table 5. Design Phase's processes, their classification, and associated artifacts

\begin{tabular}{|c|c|c|c|c|c|c|c|}
\hline \multirow[t]{2}{*}{ Design Processes } & \multicolumn{4}{|c|}{ Classification } & \multirow[t]{2}{*}{ Artifacts } & \multicolumn{2}{|l|}{ Roles } \\
\hline & 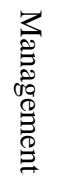 & 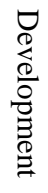 & 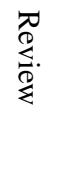 & 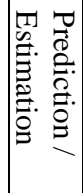 & & 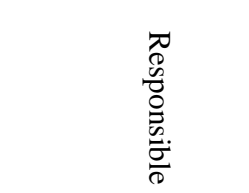 & 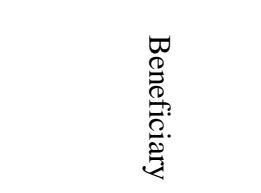 \\
\hline Handle REQ Change & - & & & & Update RTM & \multirow[t]{3}{*}{ PM } & \multirow[t]{3}{*}{$\mathrm{PT}$} \\
\hline Establish Contingency Plans & - & & & & Update PMP & & \\
\hline Establish Risk Management & - & & & & Risk Assessment & & \\
\hline Develop Security Plans & - & & & & Security Plans & Master Tester & $\begin{array}{l}\text { Testing, System } \\
\text { Team }\end{array}$ \\
\hline Develop Integration Plans & - & & & & Integration Plans & System Engineer & $\begin{array}{l}\text { Testing, System } \\
\text { Team }\end{array}$ \\
\hline Develop Architecture Design & & - & & & SDD & \multirow{4}{*}{$\begin{array}{l}\text { Architect, Master } \\
\text { Dev, DB Admin }\end{array}$} & \multirow{4}{*}{$\begin{array}{l}\text { Dev, Testing, } \\
\text { System Team }\end{array}$} \\
\hline Interface Design & & - & & & $\begin{array}{l}\text { Interface } \\
\text { Specification }\end{array}$ & & \\
\hline Database Design & & - & & & DST & & \\
\hline Develop Prototype & & - & & & Prototype & & \\
\hline Test Case Design & & - & & & UTC, ITS & Master Tester & Dev, Testing Team \\
\hline Design Review & & & - & & $\begin{array}{l}\text { Design Metrics, } \\
\text { SAR }\end{array}$ & $\begin{array}{l}\text { QA Lead, } \\
\text { Master Developer }\end{array}$ & Dev, Testing Team \\
\hline Phase Status Review & & & - & & Status Report & PM & Dev Team, PT \\
\hline $\begin{array}{l}\text { Prediction based Design } \\
\text { Metrics }\end{array}$ & & & & $\cdot$ & TAR & $\begin{array}{l}\text { QA Lead, } \\
\text { Master Tester }\end{array}$ & Testing Team \\
\hline
\end{tabular}

Table 6. Coding Phase's processes, their classification, and associated artifacts

\begin{tabular}{|c|c|c|c|c|c|c|c|}
\hline \multirow[t]{2}{*}{ Coding Processes } & \multicolumn{4}{|c|}{ Classification } & \multirow[t]{2}{*}{ Artifacts } & \multicolumn{2}{|l|}{ Roles } \\
\hline & 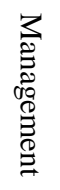 & 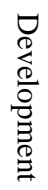 & 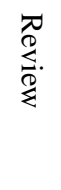 & 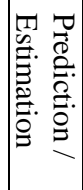 & & 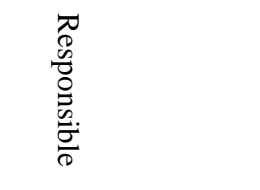 & 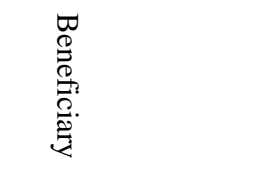 \\
\hline Handle REQ Change & - & & & & Update RTM & \multirow[t]{3}{*}{ PM } & \multirow[t]{3}{*}{$\mathrm{PT}$} \\
\hline Establish Contingency Plans & - & & & & Update PMP & & \\
\hline Establish Risk Management & - & & & & Risk Assessment & & \\
\hline Establish Dev Environment & & - & & & System Dev. Doc & Master Developer & Dev Team \\
\hline Build Release units & & - & & & Release unit & \multirow[t]{2}{*}{ Developer } & \multirow[t]{2}{*}{ Dev, Testing Team } \\
\hline Conduct Unit Testing & & - & & & Defect Log & & \\
\hline Peer Review & & & - & & $\begin{array}{l}\text { SAR, Coding } \\
\text { Metrics }\end{array}$ & $\begin{array}{l}\text { QA Lead, Master } \\
\text { Developer }\end{array}$ & Dev, Testing Team \\
\hline Phase Status Review & & & - & & Status Report & $\mathrm{PM}$ & Dev Team, PT \\
\hline $\begin{array}{l}\text { Prediction based Coding } \\
\text { Metrics }\end{array}$ & & & & - & TAR & $\begin{array}{l}\text { QA Lead, , Master } \\
\text { Tester }\end{array}$ & Testing Team \\
\hline
\end{tabular}


Table 7. Pre-Testing Phase's processes, their classification, and associated artifacts

\begin{tabular}{|c|c|c|c|c|c|c|c|}
\hline \multirow[t]{2}{*}{ Pre-Testing Processes } & \multicolumn{4}{|c|}{ Classification } & \multirow[t]{2}{*}{ Artifacts } & \multicolumn{2}{|c|}{ Roles } \\
\hline & 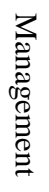 & 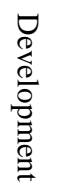 & 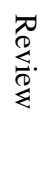 & 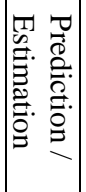 & & $\begin{array}{l}\nabla \\
8 \\
0 \\
0 \\
0 \\
0 \\
0 \\
\frac{0}{0}\end{array}$ & 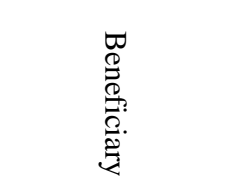 \\
\hline Establish Risk Management & - & & & & Risk Assessment & \multirow[t]{2}{*}{ PM } & \multirow[t]{2}{*}{ PT } \\
\hline Establish Contingency Plans & - & & & & Update PMP & & \\
\hline Test Case Reduction & & - & & & $\begin{array}{l}\text { Test Case } \\
\text { Repository }\end{array}$ & Master Tester & Testing Team \\
\hline $\begin{array}{l}\text { Development's Metric } \\
\text { Collection \& Analysis }\end{array}$ & & - & & & Metrics Repository & QA Lead & Testing Team \\
\hline Phase Status Review & & & - & & Status Report & PM & PT, Testing Team \\
\hline $\begin{array}{l}\text { Prediction based Reduced } \\
\text { Metrics }\end{array}$ & & & & - & TAR & $\begin{array}{l}\text { QA Lead, Master } \\
\text { Tester }\end{array}$ & Testing Team \\
\hline
\end{tabular}

Table 8. Testing Phase's processes, their classification, and associated artifacts

\begin{tabular}{|c|c|c|c|c|c|c|c|}
\hline \multirow[t]{2}{*}{ Testing Processes } & \multicolumn{4}{|c|}{ Classification } & \multirow[t]{2}{*}{ Artifacts } & \multicolumn{2}{|l|}{ Roles } \\
\hline & 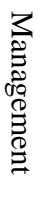 & 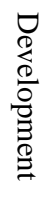 & 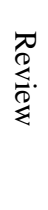 & 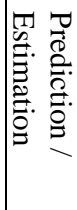 & & $\begin{array}{l}\not{0} \\
8 \\
0 \\
0 \\
0 \\
0 \\
\frac{0}{0}\end{array}$ & 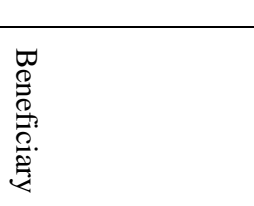 \\
\hline Establish Risk Management & - & & & & Risk Assessment & \multirow[t]{2}{*}{$\mathrm{PM}$} & \multirow[t]{2}{*}{ PT } \\
\hline Establish Contingency Plans & - & & & & Update PMP & & \\
\hline Establish Testing Environment & - & & & & STD & Master Tester & Testing Team \\
\hline Conduct integration Test & & - & & & \multirow{3}{*}{$\begin{array}{l}\text { Defect Log } \\
\text { TAR }\end{array}$} & \multirow[t]{3}{*}{ Tester } & \multirow[t]{3}{*}{ Dev, Testing Team } \\
\hline Conduct Release Testing & & - & & & & & \\
\hline Conduct Security Testing & & - & & & & & \\
\hline Phase Status Review & & & - & & Status Report & PM & $\begin{array}{l}\text { PT, Dev, Testing, } \\
\text { System Team }\end{array}$ \\
\hline
\end{tabular}

Table 9. Post-Testing Phase's processes, their classification, and associated artifacts

\begin{tabular}{|c|c|c|c|c|c|c|c|}
\hline \multirow[t]{2}{*}{ Post-Testing Processes } & \multicolumn{4}{|c|}{ Classification } & \multirow[t]{2}{*}{ Artifacts } & \multicolumn{2}{|l|}{ Roles } \\
\hline & 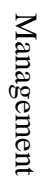 & 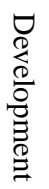 & 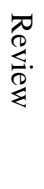 & 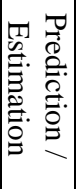 & & $\begin{array}{l}\nabla \\
0 \\
0 \\
0 \\
0 \\
0 \\
0 \\
\sigma \\
\frac{0}{0}\end{array}$ & 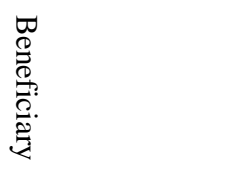 \\
\hline Establish Risk Management & - & & & & Risk Assessment & \multirow[t]{2}{*}{$\mathrm{PM}$} & \multirow[t]{2}{*}{ PT } \\
\hline Establish Contingency Plans & - & & & & Update PMP & & \\
\hline Plan for Deployment & - & & & & $\begin{array}{l}\text { Training Plans, } \\
\text { User Manual }\end{array}$ & System Engineer & $\begin{array}{l}\text { Customer, Dev., } \\
\text { Testing Team }\end{array}$ \\
\hline Conduct Acceptance Test & & - & & & TAA & Master Tester, User & System Testing \\
\hline $\begin{array}{l}\text { Complete Release } \\
\text { Documentation }\end{array}$ & & - & & & $\begin{array}{l}\text { Release } \\
\text { Documentation }\end{array}$ & Testing Team & All Stakeholders \\
\hline Phase Status Review & & & - & & Status Report & $\mathrm{PM}$ & PT, Testing Team \\
\hline Conduct Reliability Estimation & & & & - & Readiness Report & $\begin{array}{l}\text { QA Lead, } \\
\text { Master Tester }\end{array}$ & $\begin{array}{l}\text { Customer, Dev., } \\
\text { Testing Team }\end{array}$ \\
\hline
\end{tabular}


Table 10. Deployment and Operation Phase's processes, their classification, and associated artifacts

\begin{tabular}{|c|c|c|c|c|c|c|c|}
\hline \multirow{2}{*}{$\begin{array}{l}\text { Deployment and Operation } \\
\text { Processes }\end{array}$} & \multicolumn{4}{|c|}{ Classification } & \multirow[t]{2}{*}{ Artifacts } & \multicolumn{2}{|l|}{ Roles } \\
\hline & 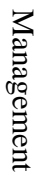 & 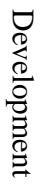 & $\begin{array}{l}\underset{0}{0} \\
\stackrel{0}{0} \\
\stackrel{0}{<}\end{array}$ & 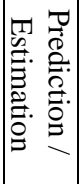 & & 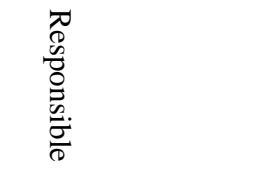 & 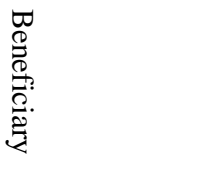 \\
\hline Establish Risk Management & - & & & & Risk Assessment & \multirow[t]{2}{*}{$\mathrm{PM}$} & \multirow[t]{2}{*}{ PT } \\
\hline Establish Contingency Plans & - & & & & Update PMP & & \\
\hline $\begin{array}{l}\text { Deploy Release in Operational } \\
\text { Environment }\end{array}$ & & $\cdot$ & & & Complete Release & Technical Support & System Testing \\
\hline Conduct Training Plans & & - & & & \multirow{4}{*}{$\begin{array}{l}\text { RTR } \\
\text { USRR }\end{array}$} & \multirow[t]{4}{*}{ User, System Team } & \multirow[t]{4}{*}{ All Stakeholders } \\
\hline Support Release Operation & & - & & & & & \\
\hline Review Release Security & & & - & & & & \\
\hline Review Release Performance & & & $\square$ & & & & \\
\hline
\end{tabular}

\section{CONCLUSION}

The framework introduces a completely integrated view of the development activities and their related artifacts treating the testing and QA practices as integral parts of the development process.

The framework promotes the efficiency of the stakeholders through the redistribution of their roles in the different teams. It introduces a new role relating the managerial and technical interest at development and testing levels to find a linkage between members of different teams in the project without the overlap in their roles, So that teams work all harmoniously tight proceeding according to system administrative work tightly in line with the plan developed for the project as a whole.

We aim to touch in our future studies the construction of a software quality improvement model based on the measurement analysis at various levels of the proposed activities classification's dimensions.

\section{REFERENCES}

[1] Yu BengLeau, WooiKhong Loo Wai Yip Tham, and Soo Fun Tan, "Software Development Life Cycle AGILE vs. Traditional Approaches", International Conference on Information and Network Technology (ICINT), 2012, pp. 162-167.

[2] Shikhamaheshwari, Dinesh Ch. Jain, "A Comparative Analysis of Different types of Models in Software Development Life Cycle", International Journal of Advanced Research in Computer Science and Software Engineering, Vol.2, No.5, 2012, pp.285-290.

[3] Kirti Nagpal,Raman Chawla, "Improvement of Software Development Process: A new SDLC Model", International Journal of Latest Research in Science and Technology, Vol. 1, No.3, 2012, pp.217-224.

[4] T.Rajani Devi, "Importance of Testing in Software Development Life Cycle", International Journal of Scientific \& Engineering Research, Vol.3, No.5, 2012, pp.1-5.

[5] Jamie S. Gordon, Robert F. Roggio, " A Comparison of Software Testing Using the Object-Oriented Paradigm and Traditional Testing", Proceedings of the Conference for Information Systems Applied Research, San Antonio, Texas, USA, Vol. 6, No.28, 2013.
[6] Rashdeep Kaur, Sunil Gulati," A framework for Analyzing Software Quality using Hierarchical Clustering", International Journal on Computer Science and Engineering (IJCSE), Vol.3, No.2, 2011, pp.854860.

[7] T.M.H koshgoftaar, P.Rebours and N.seliya," Software Quality analysis by combining multiple projects and learners", software Quality Journal, Vol.17, 2009, pp.2549.

[8] S.Shafi,S.M.Hassan,A.Arshaq,M.J.Khan,S.Shamail, "Software Quality Prediction Techniques: A comparative Analysis ", $4^{\text {th }}$ International Conference on Emerging Technologies (ICET),, 2008, pp.242-246.

[9] Heena Kapila, Satwinder singh, "Analysis of CK Metrics to predict Software Fault-Proneness using Bayesian Inference", International Journal of Computer Applications (IJCA), Vol. 74, No. 2, 2013, pp. 1-4.

[10] Milad Hanna, Nahla El-haggar and Mostafa Sami. Article: Reducing Testing Effort using Automation", International Journal of Computer Applications, Vol. 81, No. 8, 2013, PP.16-21.

[11] Hilda B. Klasky, "A study of Software Mertics", M.S thesis, Department of Electrical and Computer Engineering, Rutgers University, New Brunswick, New Jersey, 2003.

[12] Saddam H. Ahmed, Taysir Hassan A. Soliman , and Adel A. Sewisy, " A Hybrid Metrics Suite for Evaluating Object-Oriented Design", International Journal of Software Engineering, Vol. 6, No. 1, 2013, pp. 65-82.

[13] D. Jeyamala, S. Balamurugan, A. Jalila, K. Sabari Nathan, " Fault-prone Components Identification for Real Time Complex Systems Based on Criticality Analysis", International Journal of Computer Science and Informatics, Vol. 3, No. 2, 2013, pp. 17-23.

[14] Gurvinder Singh, Baljit Singh Saini , Neeraj Mohan, " A Systematic Literature Review on Software Fault Prediction based on Qualitative and Quantitative Factors", International Journal on Advanced Computer Theory and Engineering (IJACTE), Vol.2, No. 2, 2013, pp. 47-50. 
[15] Shaik Nafeez Umar et al, "Software Testing Defect Prediction Model -A Practical Approach", International Journal of Research in Engineering and Technology (IJRET), Vol.2, No.5, 2013, pp. 741-745.

[16] Menka Gupta, PratimaGautam, " A Novel Approach for Identifying Software Fault Prediction in mining", International Journal of Technological Exploratoration and Learning (IJTEL), Vol.2, No.6, 2013, pp. 341-343.

[17] Nirvikar Katiyar, Raghuraj Singh, " Prediction of Number of Faults And Time To Remove Errors", International Journal of Computational Engineering Research, Vol.3, No.4, 2013, pp. 57-65.

[18] S.Kim, T.Zimmermann, E.J.W.Jr., and A.Zeller, " Predicting faults from cached history", $29^{\text {th }}$ International Conference on Software Engineering (ICSE), 2013, pp.489-498.

[19] T.J.Ostrand, E.J.Weyuker, and R.M.Bell, " Predicting the location and number of faults in large software system", IEEE Transactions on Software Engineering, Vol.31, No.4, 2005, pp. 340-355.

[20] Anshu Bansal, Sudhir Pundir, " A Review On Approaches and Models Proposed for Software Reliability Testing", International Journal of Computer \& Communication Technology, Vol.4, No.2, 2013, pp. 7-9.

[21] J Prabhu and N Malmurugan, "Article: A Model for GUI Automated Testing Framework in Software System", International Journal of Computer Applications, Vol.64, NO.15, 2013,PP. 16-20.

[22] Rakesh Roshan, RabinsPorwal, Chandra Mani Sharma, "Review of Search Techniques in Software Testing ", international Journal of Computer Applications, Vol.51, No.6, 2012, pp.42-45.

[23] Dinesh Kumar Saini., "Article: Software Testing for Embedded Systems", International Journal of Computer Applications, Vol.43,No.17,2012,PP.1-6 .

[24] Anuja Jain M, Swarnalatha P, Muhammad Rukunuddin Ghalib and S Prabu. Article: Web-Based Automation Testing Framework", International Journal of Computer Applications, Vol. 45, No. 16, 2012, PP.1-5.

[25] Anudeep Ediga, " High Accuracy Prediction Framework for Predicting Defect Prone Software Components", International Journal of Information and Computation Technology, Vol.3,No.10, 2013, pp.985-992.
Table 11. Appendix A - Abbreviations

\begin{tabular}{|l|l|}
\hline AUT & Acceptance User Test \\
\hline BA & Business Analyst \\
\hline DST & Data Storage Design \\
\hline GUI & Graphical User Interface \\
\hline HRM & Human Resource Manager \\
\hline ITS & Integration Test Script \\
\hline MTP & Master Test Plan \\
\hline PM & Project Manager \\
\hline PMP & Project Management Plan \\
\hline PSD & Project Scope Document \\
\hline PT & Planning Team \\
\hline QA & Quality Assurance \\
\hline RAM & Responsibilities Matrix \\
\hline RTM & Requirement Traceability Matrix \\
\hline RTR & Release Troubles Report \\
\hline SAR & Static Analysis Report \\
\hline SDD & System Design Document \\
\hline SRD & System Requirement Document \\
\hline STD & System Testing Document \\
\hline TAA & Test Analysis Approval \\
\hline TAR & Test Analysis Report \\
\hline URD & User Requirement Document \\
\hline USRR & User Satisfaction Review Report \\
\hline UTC & User Test Case \\
\hline WBS & Work Breakdown Structure \\
\hline
\end{tabular}

